\title{
Multi-component BEM for the Helmholtz equation: A normal derivative method
}

\author{
H.A.M. Ben Hamdin and G. Tanner* \\ School of Mathematical Sciences, University of Nottingham, Nottingham, UK
}

\begin{abstract}
We describe a multi-component boundary element method for predicting wave energy distributions in a complex built-up system with material properties changing discontinuously at boundaries between sub-components. We point out that depending on the boundary conditions and the number of interfaces between sub-components, it may be advantageous to use a normal derivative method to set up the integral kernels. We describe how the resulting hypersingular integral kernels can be regularised. The method can be used to minimise the number of weakly singular integrals thus leading to BEM formulations which are easier to handle.
\end{abstract}

Keywords: Multi-component boundary element method, hypersingular integral, Helmholtz equation, Green function

\section{Introduction}

Determining the wave energy distribution in complex built-up structures for which material properties change from one sub-structure to the next is a common problem in mechanical engineering, acoustics, optics and quantum mechanics. The vibration dynamics of coupled plates with different thickness or electromagnetic waves in a homogeneous media with discontinuous changes in the dielectric constants are examples thereof. An efficient tool to treat such problems is the Boundary Element Method (BEM) [1-3] making use of the fact that the free Green function is known in each sub-domain. It has advantages over other popular numerical techniques such as the Finite Element Method (FEM) [4] or the Finite Difference Method (FDM) [5] due to the reduction of the dimensionality of the problem by one.

In the following, we will restrict our analysis to the two-dimensional Helmholtz equation, although the concepts and ideas are of use to other wave equations and in higher dimensions. Owing to the singularity of the Green function, some care is needed when setting up the Boundary Integral Equations (BIEs) to treat singular or even hypersingular integrals. Regularisation processes together with a careful treatment of the numerical discretisation have made the BEM an efficient and generally well-conditioned numerical solution procedures. The hypersingular terms often arise when taking normal derivatives of the integral equation. A regularisation formula for the hypersingular integrals was first obtained by Maue [6] in 1949, and has been rederived in a simpler way by Mitzner [7] in 1966 for acoustic scattering from curved interfaces between two media of different material densities. Furthermore, Kutt [8] obtain the same formula for scattering problems in two and three dimensions by adopting the finite part of the hypersingular integrals. Burton and Miller [9] used Maue's formula to treat hypersingular integral in the context of finding unique solutions for wave radiation and scattering problems.

In principle, there are two ways to regularise hypersingular terms in the BIE. The first approach aims at removing all singularities analytically, whereas in the second approach, the regularisation is completed after discretisation where divergent terms cancel by using a Galerkin or collocation formulation [10,11]. Krisnasamy et al. [12] dealt

\footnotetext{
*Corresponding author: G. Tanner, School of Mathematical Sciences, University of Nottingham, Nottingham NG7 2RD, UK. E-mail: Gregor. Tanner@nottingham.ac.uk.
} 
with the hypersingularity for an acoustic wave scattering problem; their method is free of any discretisation assumption, it demands only sufficient smoothness on the density function at the singularity. Kutt [8] developed a numerical approach to evaluate one-dimensional hypersingular integrals in finite part representation using Gaussian quadrature. Tanaka et al. and Sladek $[10,11]$ give a survey on the treatments of all types of singularities.

For multi-component systems with discontinuous changes of the wave speed across interfaces, the free Green function is often only known for individual sub-components, but not for the fully composed system (This is the case, if material parameters change only at interfaces but are constant otherwise). Likewise, for a BEM analysis of a system with a complex shape it is often advantageous to simplify the domain by decomposing it into sub-components. The BIEs for each sub-component are then coupled through compatibility and equilibrium conditions along the interfaces between the sub-components. This procedure is called a multi-component BEM treatment [13-15]. Since the sub-component technique needs new boundary elements along the interfaces, this results in a large number of unknowns and thus a larger system of equations. So for large problems, parallel computing is often desirable in which the boundary element analysis is performed for each sub-component iteratively [16,17]. This is known as the domain decomposition method (DDM) [17]. Sugino et al. [18] demonstrated the usefulness of a BEM analysis with DDM for the interface motion of a two-layer fluid.

In this paper we consider the special situation, where we have Dirichlet Boundary Conditions (DBCs) with a vanishing wave function at the outer boundary. These boundary conditions are typical for plate or membrane problems with clamped or fixed boundaries. In the single domain case, it is then advantageous to formulate the BIEs by taking an additional normal derivative of the integral kernel. One can in this way eliminate weak (logarithmic) singularities in the integrands and there is no need for further regularisations of the integral kernel (such as Telles regularisation [19]). However, for a multi-component BEM, this leads to second order derivatives of the integral kernels at interfaces which need to be regularised as mentioned above. The resulting integral equations may then again contain weakly singular terms. We show in this paper how a normal derivative multi-component BEM is constructed and argue that depending on the length of the boundary compared to the length of the interfaces, it may be useful to use such a normal derivative method to minimise the use of additional regularisation techniques. In particular, we derive analytic expression leading to a regularisation of the singular BIEs.

The paper is structured as follows: Section 2 describes the types of problems we are dealing with. The explicit form of the BIEs are presented in Section 3. In Section 4, we derive an integral identity for the hypersingular integral. We demonstrate that this facilitates the numerical evaluation of the integral equations for some example problems in Section 5.

\section{The general setting of the wave problem}

As a starting point, we will consider a two-dimensional domain $\Omega$ with two sub-components such as shown in Fig. 1.

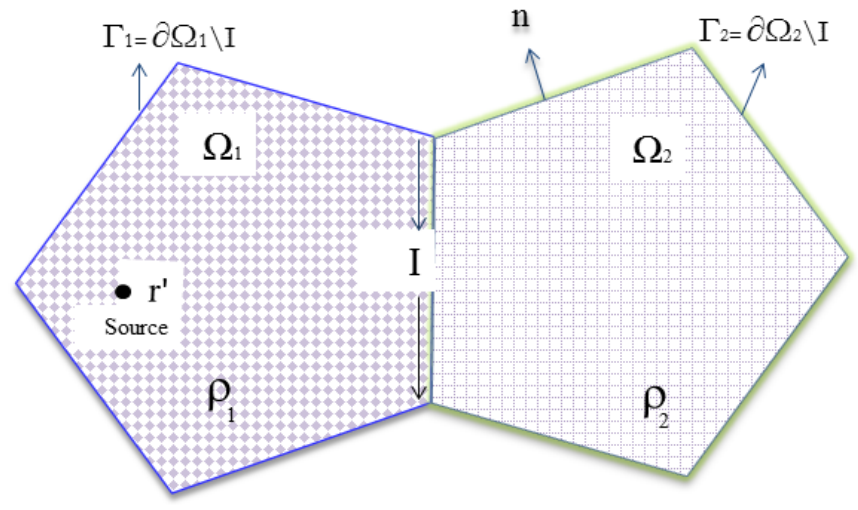

Fig. 1. Geometric configuration of the coupled domains. 
We assume that the configuration $\Omega=\Omega_{1} \cup \Omega_{2}$ consists of two components with different material parameters. That is, the left sub-component $\Omega_{1}$ and the right sub-component $\Omega_{2}$ are separated by, for example, a change of material densities $\rho_{1}$ and $\rho_{2}$ at the interface and the wave speed and absorption coefficients change discontinuously at the interface. Here, we do not restrict ourselves to specific applications. The Helmholtz equation for domains with different parameters describes many physical phenomena such as a quantum particle in finite domain with discontinuous step potential, an electromagnetic cavity with discontinuous refractive index, acoustic cavities of different materials for example air/solid or fluid/solid or coupled membrane problems.

We aim to numerically calculate the Green function $G\left(\mathbf{r}, \mathbf{r}^{\prime} ; k\right)$ which solves the inhomogeneous Helmholtz equation,

$$
\left(\nabla_{\mathbf{r}}^{2}+k_{j}^{2}\right) G\left(\mathbf{r}, \mathbf{r}^{\prime} ; k_{j}\right)=-\delta\left(\mathbf{r}-\mathbf{r}^{\prime}\right)
$$

where $G\left(\mathbf{r}, \mathbf{r}^{\prime} ; k_{j}\right)$ describes the amplitude of the wave field in the interior of $\Omega_{j}$, and $\mathbf{r}$ and $\mathbf{r}^{\prime}$ are the observation and the source points. The source excitation is represented by the two-dimensional $\delta$-distribution. The wavenumber is defined as

$$
k_{j}=\frac{\omega}{c_{j}}+\imath \eta_{j}, \quad \eta_{j}>0, \quad \imath=\sqrt{-1}, \quad j=1,2
$$

and $\omega$ is the angular frequency. The constants $c_{j}$ and $\eta_{j}$ are respectively the wave velocity and the damping parameter. We shall denote the external boundary for each sub-component by,

$$
\Gamma_{j}=\partial \Omega_{j} \backslash I, \quad I=\partial \Omega_{1} \cap \partial \Omega_{2}
$$

where $I$ denotes the internal boundary (interface).

In what follows, we consider DBCs along the external boundaries $\Gamma_{j}$, that is,

$$
G_{j}\left(q, \mathbf{r}^{\prime} ; k_{j}\right)=0, \quad q \in \Gamma_{j}
$$

The boundary condition at the interface is given by the continuity of the Green function and the equilibrium of the energy flux in a direction normal to the interface; these can be expressed as

$$
\left.\begin{array}{c}
G_{1}\left(q, \mathbf{r}^{\prime} ; k_{1}\right)=G_{2}\left(q, \mathbf{r}^{\prime} ; k_{2}\right) \\
\rho_{2} \frac{\partial G_{1}\left(q, \mathbf{r}^{\prime} ; k_{1}\right)}{\partial n_{q}}=\rho_{1} \frac{\partial G_{2}\left(q, \mathbf{r}^{\prime} ; k_{2}\right)}{\partial n_{q}}
\end{array}\right\}, \quad q \in I
$$

where $\partial / \partial n_{q}$ denotes the outward normal derivative at the boundary point $q$. The constants $\rho_{1}$ and $\rho_{2}$ are the material densities in the left and right sub-domain, respectively. The wavenumbers $k$ 's and material densities $\rho$ 's are related by,

$$
\frac{\rho_{1}}{\rho_{2}}=\frac{k_{1}^{2}}{k_{2}^{2}} .
$$

\section{Derivation of the boundary integral equations}

In this section we derive the coupled BIEs for the components $j=1,2$. The free Green function $G_{0}\left(\mathbf{r}, \mathbf{r}^{\prime} ; k\right)$ for the two-dimensional Helmholtz equation has the form, 


$$
G_{0}\left(\mathbf{r}, \mathbf{r}^{\prime} ; k\right)=\frac{l}{4} H_{0}^{(1)}\left(k\left|\mathbf{r}-\mathbf{r}^{\prime}\right|\right)
$$

where $H_{0}^{(1)}$ is the 0 -th order Hankel function of the first kind. Note that $G_{0}\left(\mathbf{r}, \mathbf{r}^{\prime}\right)$ satisfies the inhomogeneous Helmholtz Eq. (1), but not necessarily the boundary conditions Eq. (2). In the following, we assume that the source is located in the left sub-component, i.e, $\mathbf{r}^{\prime} \in \Omega_{1}$. After applying Eq. (1) to both $G_{0}(\mathbf{q}, \mathbf{r})$ and $G_{j}\left(\mathbf{q}, \mathbf{r}^{\prime}\right)$, multiplying with $G_{j}$ and $G_{0}$ from the left and subtracting the resulting equations as well as integrating over the domain $\Omega_{j}$, one has

$$
\begin{aligned}
& \iint_{\Omega_{j}}\left[G_{0}(\mathbf{q}, \mathbf{r}) \nabla^{2} G_{j}\left(\mathbf{q}, \mathbf{r}^{\prime}\right)-G_{j}\left(\mathbf{q}, \mathbf{r}^{\prime}\right) \nabla^{2} G_{0}(\mathbf{q}, \mathbf{r})\right] \mathrm{d} A_{\mathbf{q}}= \\
& -\iint_{\Omega_{j}}\left[\delta_{1 j} G_{0}(\mathbf{q}, \mathbf{r}) \delta\left(\mathbf{q}-\mathbf{r}^{\prime}\right)-G_{j}\left(\mathbf{q}, \mathbf{r}^{\prime}\right) \delta(\mathbf{q}-\mathbf{r})\right] \mathrm{d} A_{\mathbf{q}}, \quad j=1,2 .
\end{aligned}
$$

Using Green's second identity and partitioning the resulting integrals by writing the boundary of each sub-component as $\partial \Omega_{j}=\Gamma_{j} \cup I$, one obtains,

$$
\begin{aligned}
& \int_{I}\left[G_{0}(q, \mathbf{r}) \frac{\partial G_{j}^{I}\left(q, \mathbf{r}^{\prime}\right)}{\partial n_{q}}-G_{j}^{I}\left(q, \mathbf{r}^{\prime}\right) \frac{\partial G_{0}(q, \mathbf{r})}{\partial n_{q}}\right] \mathrm{d} q \\
& +\int_{\Gamma_{j}} G_{0}(q, \mathbf{r}) \frac{\partial G_{j}\left(q, \mathbf{r}^{\prime}\right)}{\partial n_{q}} \mathrm{~d} q=-\delta_{1 j} G_{0}\left(\mathbf{r}, \mathbf{r}^{\prime}\right)+G_{j}\left(\mathbf{r}, \mathbf{r}^{\prime}\right),
\end{aligned}
$$

where we have already implemented the DBCs (Eq. (2)) on $\Gamma_{j}$. Note that the integrands in Eq. (7) are bounded as long as $\mathbf{r}$ is an interior point. Difficulties may arise when considering the limit $\mathbf{r} \rightarrow \Gamma_{j}$ or $I$. In Eq. (7), for example, $G_{0}$ has a logarithmic singularity for $\mathbf{r} \rightarrow q$ and

$$
\lim _{\mathbf{r} \rightarrow \boldsymbol{\beta}} \partial G_{0}(q, \mathbf{r}) / \partial n_{q}=\partial G_{0}(q, \beta) / \partial n_{q}+\frac{1}{2} \delta(q-\beta)
$$

with $\beta \in \partial \Omega$, known also as the jump relation [20]. Note that $\partial G_{0}(q, \beta) / \partial n_{q}$ is regular at $q=\beta$. The weakly singular terms due to $G_{0}(q, \beta)$ can be regularised using, for example, a Telles transformation [19]. For a single domain cavity with DBCs, a well known trick to avoid this additional complication is to take the normal derivative of the BIE with respect to $\mathbf{r}$ (or $\beta$ ) transforming all $G_{0}$ terms to $\partial G_{0} / \partial n_{\beta}$ terms. These can in turn be handled by the jump relation, Eq. (8), which clearly separates the regular and singular part.

We propose to extend this idea to multi-component methods with DBCs on the outer boundaries. That is, we proceed by taking the normal derivative of Eq. (7) with respect to $\mathbf{r}$. We then let the interior point $\mathbf{r}$ approach the boundary point $\beta$, that is, applying the operator $\vec{n}_{\beta} \cdot \nabla_{\mathbf{r}}$ to both sides of the BIE Eq. (7).

Here, we need to distinguish two cases, that is, $\beta \in \Gamma_{j}$, and $\beta \in I$. If $\beta \in \Gamma_{j}$, we are able to exchange the order of differentiation and integration for the second integral in Eq. (7) using the classical jump relation, Eq. (8). One obtains

$$
\begin{aligned}
& \int_{I}\left[\frac{\partial G_{0}(q, \beta)}{\partial n_{\beta}} \mu_{j}^{I}\left(q, \mathbf{r}^{\prime}\right)-G_{j}^{I}\left(q, \mathbf{r}^{\prime}\right) \frac{\partial^{2} G_{0}(q, \beta)}{\partial n_{\beta} \partial n_{q}}\right] \mathrm{d} q+\int_{\Gamma_{j}} \frac{\partial G_{0}(q, \beta)}{\partial n_{\beta}} \mu_{j}\left(q, \mathbf{r}^{\prime}\right) \mathrm{d} q \\
& +\frac{\mu_{j}\left(\beta, \mathbf{r}^{\prime}\right)}{2}=\mu_{j}\left(\beta, \mathbf{r}^{\prime}\right)-\delta_{1 j} \frac{\partial G_{0}\left(\beta, \mathbf{r}^{\prime}\right)}{\partial n_{\beta}}, \quad \beta \in \Gamma_{j}
\end{aligned}
$$

where 


$$
\mu_{j}\left(\beta, \mathbf{r}^{\prime}\right)=\lim _{\mathbf{r} \rightarrow \beta} \frac{\partial G_{j}\left(\mathbf{r}, \mathbf{r}^{\prime}\right)}{\partial n_{\beta}} .
$$

For the case $\beta \in I$, we are not able to interchange the order of differentiation and integration in the second integral in Eq. (7), because the integrand develops a $1 /(\beta-q)^{2}$ singularity (hypersingular). Similar to Cauchy's principal value integral, the regularised integral is defined by taking a special limit, i.e by excluding the infinite part that contains the singularity,

$$
\frac{\partial}{\partial n_{\beta}} \int_{I} \frac{\partial G_{0}(q, \beta)}{\partial n_{q}} \mathrm{~d} q=\lim _{\mathbf{r} \rightarrow \beta} \int_{I} \frac{\partial^{2} G_{0}(q, \mathbf{r})}{\partial n_{\beta} \partial n_{q}} \mathrm{~d} q \neq \int_{I} \lim _{\mathbf{r} \rightarrow \beta} \frac{\partial^{2} G_{0}(q, \mathbf{r})}{\partial n_{\beta} \partial n_{q}} \mathrm{~d} q
$$

It should be noted that when $\beta \in I$, the 'limit of the integral $\neq$ the integral of the limit'. To avoid the hypersingular integral above one needs to take the limits properly and then do the numerical evaluation. We will explain in the next section, how non-singular expressions for the integrals in (Eq. (10)) are obtained.

\section{Regularisation of the hypersingular integral}

The existence of the hypersingular integral is determined by the behaviour of the integrand in the neighbourhood of the singular point. We can thus restrict ourselves to an analysis of the interval of length $\Delta q$ around $q=\beta$, where $\Delta q$ is the length of a boundary element. For the two-dimensional Green function, Eq. (5), using relations between Hankel functions of different order and their derivatives, one obtains the following relation,

$$
\lim _{\Delta \mathbf{r} \rightarrow 0} \int_{0}^{\Delta q} \frac{\partial^{2}}{\partial n_{\beta} \partial n_{q}} G_{0}(q, \mathbf{r}) \mathrm{d} q=\frac{l}{4} \lim _{\Delta r \rightarrow 0}\left\{k^{2} \int_{0}^{\Delta q} H_{0}^{(1)}(k \rho) \mathrm{d} \sigma+\int_{0}^{\Delta q}-\frac{\partial}{\partial q}\left[k \frac{\sigma}{\rho} H_{1}^{(1)}(k \rho)\right] \mathrm{d} \sigma\right\}
$$

where $\Delta r=|\mathbf{r}-\mathbf{r}(\beta)|, \sigma=|\beta-q|$, and $\rho=\sqrt{(\Delta r)^{2}+\sigma^{2}}$ as depicted in Fig. 2. (For simplicity, we adopt the notation where $\beta$ and $q$ stand for both the parametrisation of the boundary and the vector representing the point in two dimension on the boundary.)

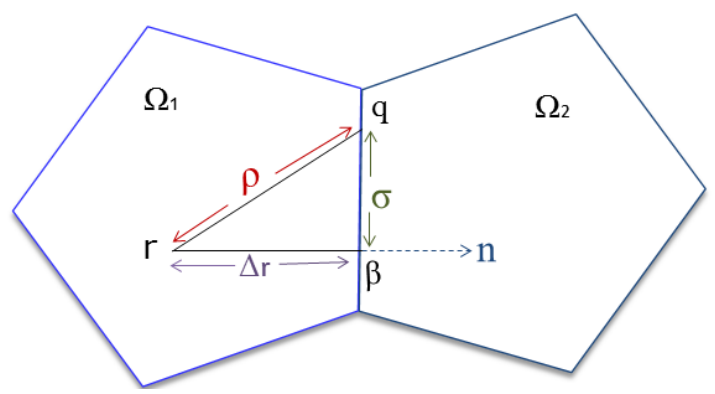

Fig. 2. Geometrical illustration of an interior point $\mathbf{r}$ approaching a boundary point $\beta \in \partial \Omega, \rho=|\mathbf{r}-\mathbf{r}(q)|, \sigma=|\mathbf{r}(\beta)-\mathbf{r}(q)|$ and $\Delta \mathbf{r}=|\mathbf{r}-\mathbf{r}(\beta)|$.

The first integral in the RHS of Eq. (11) has a weak (integrable) singularity of the order $O(\ln \rho)$.

So taking the limit commutes with doing the integration. This is not the case with the second integral due to the hypersingular kernel; if one takes the limit before doing the integration, one has

$$
\int_{0}^{\Delta q} \lim _{\Delta \mathbf{r} \rightarrow 0}\left[-\frac{\partial}{\partial q}\left[k \frac{\sigma}{\rho} H_{1}^{(1)}(k \rho)\right]\right] \mathrm{d} \sigma=\int_{0}^{\Delta q}-\frac{\partial}{\partial q}\left[k \frac{\sigma}{\sigma} H_{1}^{(1)}(k \sigma)\right] \mathrm{d} \sigma=\left[k H_{1}^{(1)}(k \sigma)\right]_{0}^{\Delta q} \rightarrow \infty
$$


which is diverging when the argument of the Hankel function tends to zero. Evaluating the integral first and then taking the limit leads to the non-singular expression $\left[6^{-9,21]}\right.$

$$
\lim _{\Delta r \rightarrow 0} \int_{\beta}^{\beta+\Delta q} \frac{\partial^{2} G_{0}(q, \mathbf{r})}{\partial n_{\beta} \partial n_{q}} \mathrm{~d} q=k^{2} \int_{\beta}^{\beta+\Delta q} G_{0}(q, \beta) \mathrm{d} q+\frac{\partial}{\partial q} G_{0}(\beta+\Delta q, \beta)
$$

It may be of interest to illustrate the integral identity (Eq. (12)) by sketching the hypersingular kernel in the limit $\Delta r \rightarrow 0$. Figure 3 shows that when $\Delta r=0$, the real part becomes singular. By considering small $\Delta r>0$, one observes that the area above the x-axis, region $A_{1}$ in Fig. 3 , is cancelled by the corresponding area below the x-axis, region $A_{2}$. Hence, the integrals remain finite and the limit $\Delta r \rightarrow 0$ exists. Clearly, changing the limit and integration leads to singular behaviour.

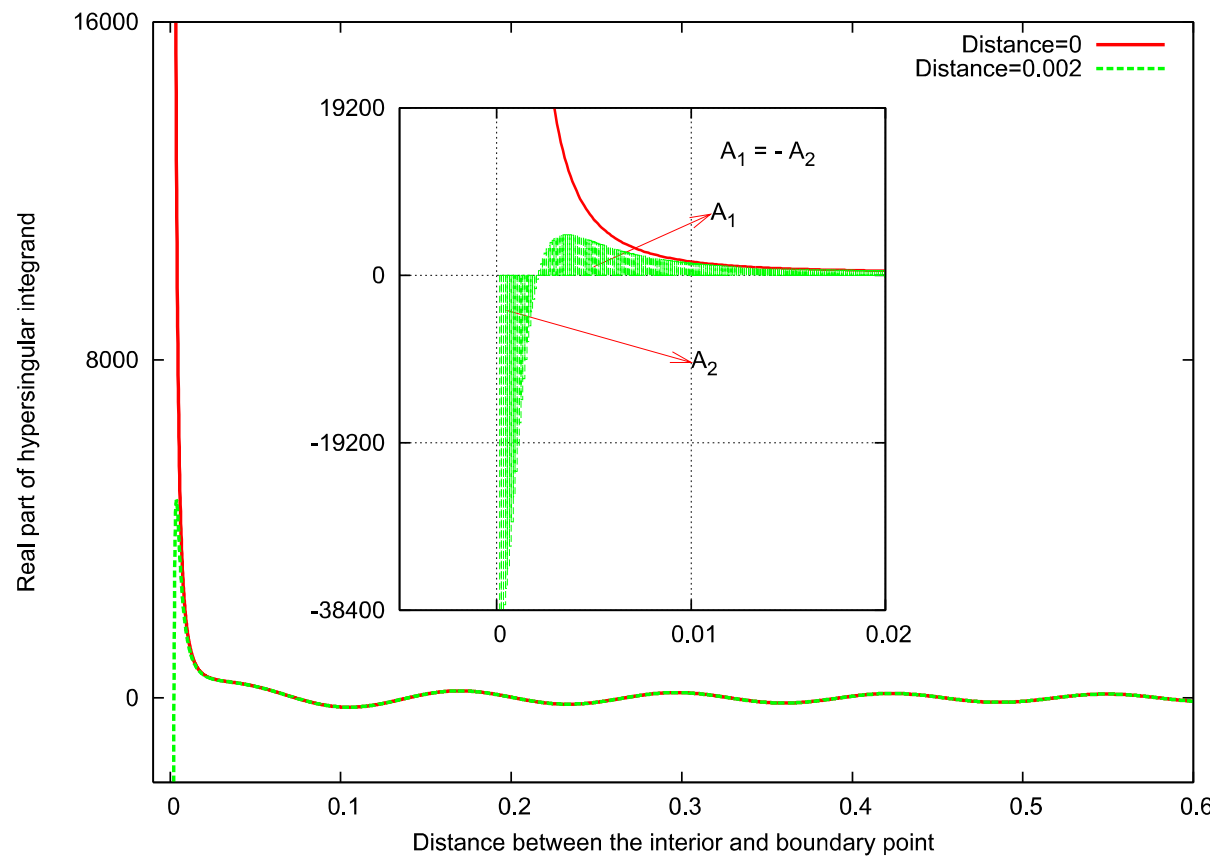

Fig. 3. Real part of the hypersingular integrand $\mathfrak{R}\left(\frac{\partial^{2} G_{0}(q, \mathbf{r})}{\partial n_{q} \partial n_{\beta}}\right)$ plotted against the distance between the interior point $\mathbf{r}$ and the boundary point $\beta$; solid red line: $\Delta \mathbf{r}=0$; dashed, green line: $\Delta \mathbf{r}=0.002$.

Having done the regularisation, one can evaluate the original hypersingular integral term using Eq. (12), that is,

$$
\begin{aligned}
& \lim _{\Delta r \rightarrow 0} \frac{\partial}{\partial n_{\beta}} \int_{\beta}^{\beta+\Delta q} G\left(q, \mathbf{r}^{\prime}\right) \frac{\partial G_{0}(q, \mathbf{r})}{\partial n_{q}} \mathrm{~d} q=\frac{\partial}{\partial n_{\beta}} \int_{\beta}^{\beta+\Delta q}\left[G\left(q, \mathbf{r}^{\prime}\right)-G\left(\beta, \mathbf{r}^{\prime}\right)\right] \frac{\partial G_{0}(q, \beta)}{\partial n_{q}} \mathrm{~d} q \\
& +G\left(\beta, \mathbf{r}^{\prime}\right)\left\{k^{2} \int_{\beta}^{\beta+\Delta q} G_{0}(q, \beta) \mathrm{d} q+\frac{\partial}{\partial q} G_{0}(\beta+\Delta q, \beta)\right\} .
\end{aligned}
$$

The integral containing the term $\left[G\left(q, \mathbf{r}^{\prime}\right)-G\left(\beta, \mathbf{r}^{\prime}\right)\right]$ is treated using piecewise constant collocation functions. In the collocation method, a function $G$ is expressed as a histogram i.e, approximated by piecewise constant basis functions as, 


$$
G(q)=\sum_{i=1}^{N} c_{i} \psi_{i}(q),
$$

where $\psi_{i}(q)$ denotes the piecewise constant boundary element basis functions, and $N$ is the number of collocation points. Hence, by using this interpolation, the divergent term in $\left[G\left(q, \mathbf{r}^{\prime}\right)-G\left(\beta, \mathbf{r}^{\prime}\right)\right]$ Eq. (13) vanishes on the arc-length containing $\beta$.

\section{Discretisation and matrix formulations}

In this section we construct the BIEs by using the integral identity (Eq. (13)) replacing the hypersingular integrals. Therefore, for $\beta \in \Gamma_{j}$, we have the following BIEs,

$$
\begin{aligned}
& \int_{I}\left[\frac{\partial G_{0}(q, \beta)}{\partial n_{\beta}} \frac{1}{\rho_{j}} \mu^{I}\left(q, \mathbf{r}^{\prime}\right)-G^{I}\left(q, \mathbf{r}^{\prime}\right) \frac{\partial^{2} G_{0}(q, \beta)}{\partial n_{\beta} \partial n_{q}}\right] \mathrm{d} q \\
& +\int_{\Gamma_{j}}\left[\frac{\partial G_{0}(q, \beta)}{\partial n_{\beta}}-\frac{\delta(q-\beta)}{2}\right] \mu_{j}\left(q, \mathbf{r}^{\prime}\right) \mathrm{d} q=-\delta_{1 j} \frac{\partial G_{0}\left(\beta, \mathbf{r}^{\prime}\right)}{\partial n_{\beta}}
\end{aligned}
$$

with implicit implementation of the continuity conditions (Eq. (3)), and using $\rho_{2} \mu_{1}^{I}=\rho_{1} \mu_{2}^{I}=\mu^{I}$ and $G_{1}^{I}=G_{2}^{I}=G^{I}$. For $\beta \in I$, we have the following equation,

$$
\begin{aligned}
& \int_{I}\left[\frac{\partial G_{0}(q, \beta)}{\partial n_{\beta}}-\frac{\delta(q-\beta)}{2}\right] \frac{1}{\rho_{j}} \mu^{I}\left(q, \mathbf{r}^{\prime}\right) \mathrm{d} q-\lim _{\mathbf{r} \rightarrow \beta} \frac{\partial}{\partial n_{\beta}} \int_{\gamma} \frac{\partial G_{0}(q, \mathbf{r})}{\partial n_{q}}\left[G^{I}\left(q, \mathbf{r}^{\prime}\right)-G^{I}\left(\beta, \mathbf{r}^{\prime}\right)\right] \mathrm{d} q \\
& +G^{I}\left(\beta, \mathbf{r}^{\prime}\right)\left[k_{j}^{2} \int_{\gamma} G_{0}(q, \beta) \mathrm{d} q-\frac{\imath k_{j}}{2} H_{1}^{(1)}\left(\frac{k_{j} \Delta q}{2}\right)\right]-\int_{I \gamma \gamma} \frac{\partial^{2} G_{0}(q, \beta)}{\partial n_{\beta} \partial n_{q}} G^{I}\left(q, \mathbf{r}^{\prime}\right) \mathrm{d} q \\
& +\int_{\Gamma_{j}} \frac{\partial G_{0}(q, \beta)}{\partial n_{\beta}} \mu_{j}\left(q, \mathbf{r}^{\prime}\right) \mathrm{d} q=-\delta_{1 j} \frac{\partial G_{0}\left(\beta, \mathbf{r}^{\prime}\right)}{\partial n_{\beta}},
\end{aligned}
$$

where $\gamma$ denotes the interval containing the point $\beta$, that is, $\gamma=\left[\beta-\frac{\Delta q}{2}, \beta+\frac{\Delta q}{2}\right]$.

One can see that taking the normal derivative of the original BIE transfers the weakly singular terms containing $H_{0}$ from the outer boundaries to the interface. The logarithmic singularities give rise to large error terms when using the collocation method, so further regularisation is necessary. This may be achieved by a suitable coordinate transformation such as the Telles transformation [19] or by expanding the Hankel function and integrating out the logarithmically divergent term explicitly, that is,

$$
\int_{\gamma} G_{0}(q, \beta) \mathrm{d} q=\frac{l}{4}\left\{\int_{\gamma}\left[H_{0}^{1}(k|q-\beta|)-\frac{2 l}{\pi} \ln (k|q-\beta|) \mathrm{d} q\right]+\int_{\gamma} \frac{2 l}{\pi} \ln (k|q-\beta|) \mathrm{d} q\right\} .
$$

The integrand of the first integral on the RHS of Eq. (16) is non-singular and can be integrated numerically using standard Gaussian quadrature; the second integral can be integrated analytically.

The BIEs (Eqs (14) and (15)) are discretised to construct the global coefficients block matrix which form the following linear system of equations, 


$$
\left[\begin{array}{cccc}
\mathbf{K}_{\Gamma_{1} \Gamma_{1}} & \mathbf{F}_{\Gamma_{1} I} & \mathbf{S}_{\Gamma_{1} I} & \mathbf{0} \\
\mathbf{F}_{I \Gamma_{1}} & \mathbf{K}_{I I} & \mathbf{H}_{I I} & \mathbf{0} \\
\mathbf{0} & \mathbf{K}_{I I} & \mathbf{H}_{I I} & \mathbf{F}_{I \Gamma_{2}} \\
\mathbf{0} & \mathbf{F}_{\Gamma_{2} I} & \mathbf{S}_{\Gamma_{2} I} & \mathbf{K}_{\Gamma_{2} \Gamma_{2}}
\end{array}\right]\left\{\begin{array}{c}
\boldsymbol{\mu}_{1}^{\Gamma_{1}} \\
\boldsymbol{\mu}^{I} \\
\mathbf{G}^{I} \\
\boldsymbol{\mu}_{2}^{\Gamma_{2}}
\end{array}\right\}=\left\{\begin{array}{c}
\mathbf{b}_{\Gamma_{1}} \\
\mathbf{b}_{I} \\
\mathbf{0} \\
\mathbf{0}
\end{array}\right\} .
$$

This square matrix has dimension $N$ where,

$$
N=N_{\Gamma_{1}}+N_{\Gamma_{2}}+2 N_{I}, \quad j=1,2
$$

and $N_{\Gamma_{j}}$, and $N_{I}$ are the number of boundary elements on $\Gamma_{j}$, and $I$, respectively. The sub-matrices $\mathbf{K}, \mathbf{F}, \mathbf{S}$, and the source vector $\mathbf{b}$ are constructed as,

$$
\begin{aligned}
\mathbf{K}_{\Gamma_{j} \Gamma_{j}}(m, i) & =\left[2 \frac{\partial G_{0}\left(q_{m}, q_{i}\right)}{\partial n_{q_{i}}}-\delta_{m i}\right] \Delta q, \quad q_{m}, q_{i} \in \Gamma_{j} \\
\mathbf{F}_{\Gamma_{j} I}(m, i) & =2 \frac{\partial G_{0}\left(q_{m}, q_{i}\right)}{\partial n_{q_{i}}} \Delta q, \quad q_{i} \in \Gamma_{j}, q_{m} \in I \\
\mathbf{S}_{\Gamma_{j} I}(m, i) & =-2 \frac{\partial^{2} G_{0}\left(q_{m}, q_{i}\right)}{\partial n_{q_{i}} \partial n_{q_{m}}} \Delta q, \quad q_{i} \in \Gamma_{j}, q_{m} \in I \\
\mathbf{b}_{\Gamma_{1}, I} & =-2 \frac{\partial G_{0}\left(q_{i}, \mathbf{r}^{\prime}\right)}{\partial n_{q_{i}}}, \quad q_{i} \in \Gamma_{1}, I
\end{aligned}
$$

and the matrix that corresponds to the hypersingular term is given as,

$$
\mathbf{H}_{I I}(m, i)= \begin{cases}-2\left[k_{j}^{2} V(\Delta q)-\frac{\imath k_{j}}{2} H_{1}^{(1)}\left(\frac{k_{j} \Delta q}{2}\right)\right], & \text { if } q_{m}=q_{i} ; \\ \mathbf{S}_{I I}(m, i), & \text { if } q_{m} \neq q_{i},\end{cases}
$$

where $V(\Delta q)$ is given by Eq. (16). The block $\mathbf{K}_{\Gamma_{j} \Gamma_{j}}$ expresses wave contributions starting and ending on the boundary $\Gamma_{j}, \mathbf{F}_{\Gamma_{j} I}$ and $\mathbf{S}_{\Gamma_{j} I}$ contains segments starting on $\Gamma_{j}$ and hitting the interface, $\mathbf{H}_{I I}$ contains wave contributions starting and ending on the interface itself, and similarly for the other sub-matrices. The multi-component BEM leads to block-banded matrix systems with one block for each sub-component, and overlaps between blocks when sub-components have common interfaces. After solving the above linear system of equations, one obtains the full Green function by inserting the solution vector $\boldsymbol{\mu}$ into Eq. (7). This yields the Green function at any interior point in the coupled domain $\Omega$.

\section{Applications}

We applied the multi-component BEM to different geometric configurations with different combinations of material parameters. Three such example domains are depicted in Fig. 4 denoted configuration A, B and C from left to right. Configuration A and B are similar in shape, but differ in the length of the interface. The wave fields in the right and left sub-domains are thus more strongly coupled in B than in A. Configuration C consists of a regular (rectangular) domain and an irregular (polygonal) domain and it may be of interest, how the regularity of the left hand domain influences the global solution. 

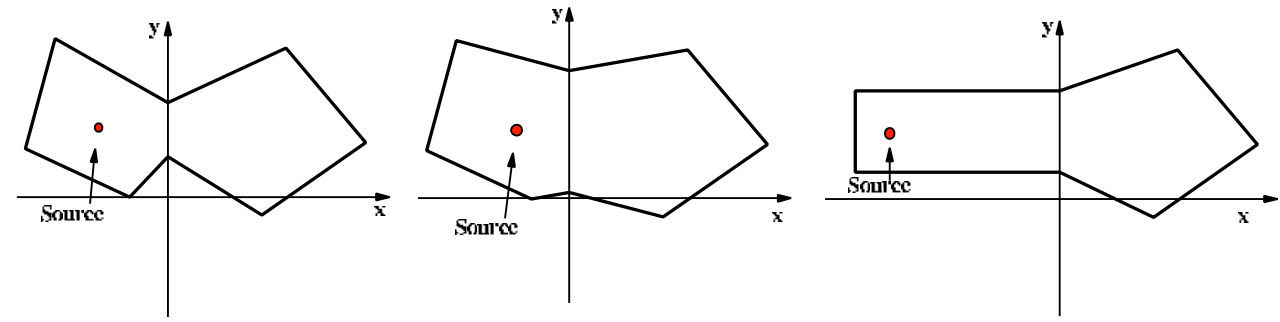

Fig. 4. From the left to the right configurations A, B and C, respectively.

(a)
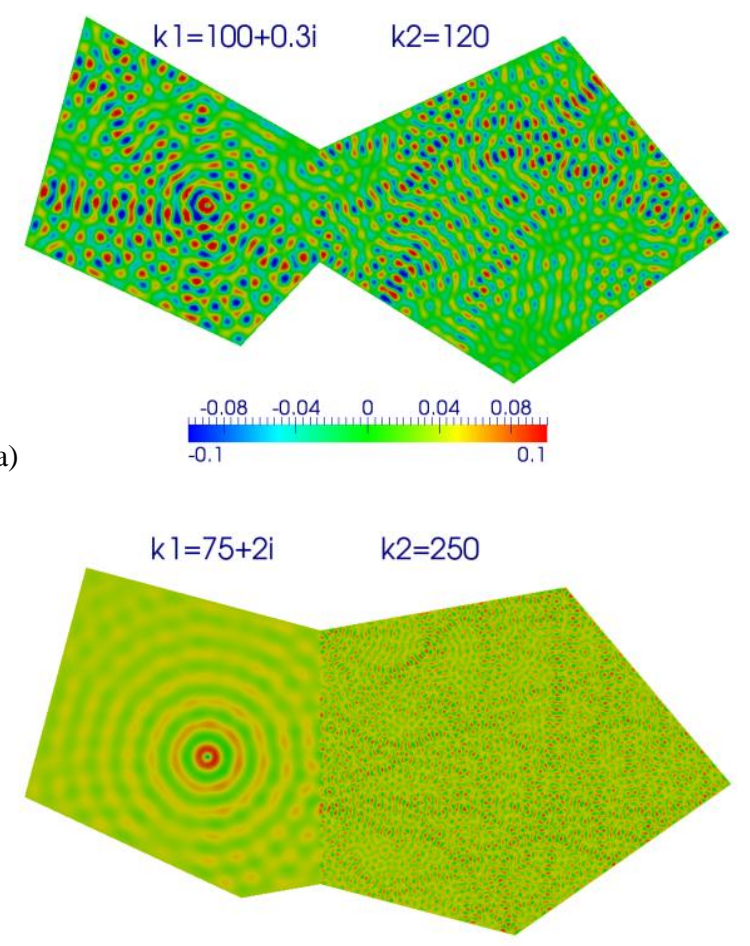

(c)
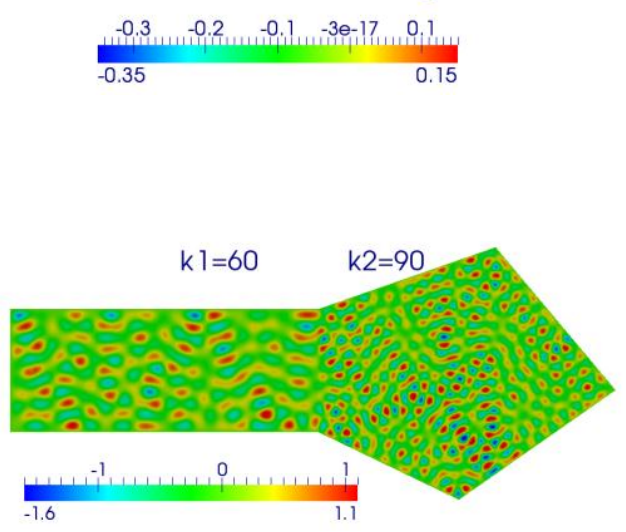

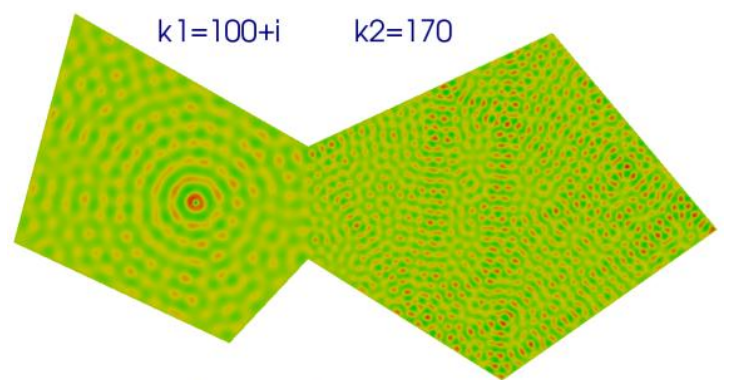

(b)

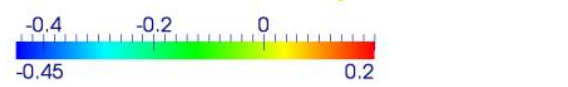

(d)

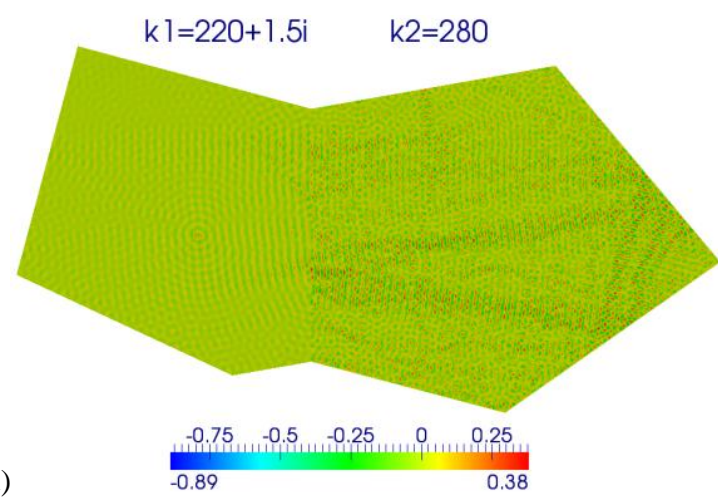

(f)

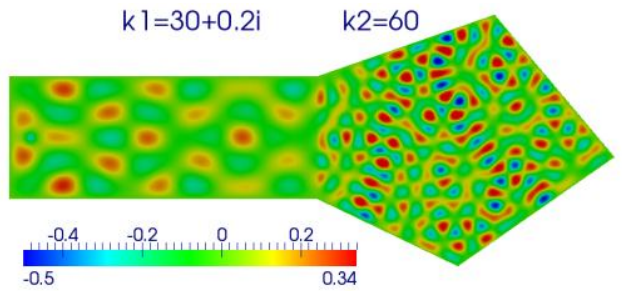

Fig. 5. $\mathfrak{R}\left(G\left(\mathbf{r}, \mathbf{r}^{\prime} ; k\right)\right)$ of configuration. A, B and $\mathrm{C}$ with different range of wavenumbers $k$, the imaginary part of $k$ represents the damping of the system 
The real part of the Green function has been plotted in Fig. 5 for the three configurations. In configuration A, Fig. 5a,b, the interface is relatively small compared to the arc-length of the boundary. Wave solutions are thus enhanced in the left domain in the case of damping. In contrast, for configuration B, Fig. 5c,d, waves easily escape to the right sub-domain, and the solutions have a more uniform amplitude distribution. In configuration C, Fig. 5e,f, regular wave patterns on the left hand side are perturbed by irregular wave solutions dominating the right hand side.

The results have been verified by comparing with FEM solutions and standard BEM (that is, not using the normal derivative method). Overall, our method performs favourable with respect to FEM and similar to standard BEM. This is to be expected as both types of BEM methods are of first order with respect to the interval length $\Delta q$; however, we find that using the normal derivative method simplifies the implementation by relying less on numerical regularisation methods.

\section{Conclusions}

We implemented a normal derivative BEM sub-components technique to investigate the wave energy distribution in multi-component systems where the wavenumber and damping parameters change discontinuously in different parts of the system. This technique can be applied where a straightforward use of classical single-domain BEM would not be possible due to the fact that the free Green function is not known across the whole domain for multi-component structures. Using the normal derivative formulation is useful as it reduces the number of regularisation procedures necessary.

\section{Acknowledgment}

HBH gratefully acknowledges the funding from the Libyan ministry of high education. GT is thankful for the funding received from the EU (FP7 IAPP grant MIDEA). The authors would like to thank Stephen Creagh for helpful discussion.

\section{References}

[1] C.A. Brebbia, J.C.F. Telles and L.C. Wrobel, Boundary element techniques, Springer-Verlag, Berlin and New York, 1984.

[2] C.A. Brebbia and S. Walker, The boundary element techniques in engineering, Newnas-Butterworths, London, 1979.

[3] G.C. Hsiao, Boundary element method-An overview, Applied Numerical Mathematics 56(2) (2006), 1356-1369.

[4] O.C. Zienkiewicz and R.L. Taylor, The finite element method, Volume I: Basic formulation and linear problems, (4th ed.), McGraw-Hill, New York, 1987; Volume II: Solid and fluid mechanics dynamics and non-linearity, McGraw-Hill, New York, 1991.

[5] E. Zauderer, Partial differential equations of applied mathematics, (3rd ed.), John Wiley and Sons, Inc., Canada, 2006.

[6] A.W. Maue, Zur Formulierung eines allgemeinen Beugungsproblems durch eine Integralgleichung, Zeitschrift für Physik A 126 (1949), 601-618.

[7] K.M. Mitzner, Acoustic scattering from an interface between Media of greatly different Density, Journal of Mathematical Physics 7(11) (1966), 2053-2060.

[8] H.R. Kutt, The numerical evaluation of principal value integrals by finite part integration, Numer Math 24 (1975), $205-210$.

[9] A.J. Burton and G.F. Miller, The application of integral equation methods to the numerical solution of some exterior boundary value problems, Proceeding of the Royal Society of London Series A 323 (1971), 201-210.

[10] M. Tanaka, V. Sladek and J. Sladek, Regularization techniques applied to Boundary element methods, Applied Mechanics Reviews 47(10) (1994), 457-499.

[11] V. Sladek and J. Sladek, Singular integrals in boundary element methods, Computational Mechanics Publications, Southampton, 1998.

[12] G. Krisnasamy, L.W. Schmerr, T.J. Rudolphi and F.J. Rizzo, Hypersingular boundary integral equations: Some applications in acoustics and elastic wave scattering, ASME Journal Applied Mechanics 5 (1990), 404-414.

[13] E. Kita and N. Kamiya, Sub-region boundary element method, JSME Int journal series A 37 (1994), 72-366.

[14] X. Lu and W.-L. Wu, A new sub-region boundary element technique based on the domain decomposition method, Engineering Analysis with Boundary Element Method 29 (2005), 944-952.

[15] P.K. Banerjee, The boundary element method in engineering, McGraw Hill Publications, London, 1994.

[16] N. Kamiya, H. Iwase and E. Kita, Parallel implementation of boundary element method with domain decomposition, Eng Anal Bound Elems 18 (1996), 209-216. 
[17] P.A. Tarek, Mathew, Domain decomposition methods for the numerical solution of partial differential equations, Lecture notes in computational science and engineering 61, Springer-Verlage, Berlin, 2008.

[18] R. Sugino, H. Imai and N. Tosaka, Boundary element scheme with domain decomposition approach for moving interface phenomenon, Proceedings 12th international conference on domain decomposition methods, 2001.

[19] J.C.F. Telles, A self adaptive coordinate transformation for efficient numerical evaluation of general boundary element integrals, International Journal for Numerical Methods in Engineering 24(4) (1987), 959-973.

[20] W. Hackbusch, Integral equations: Theory and numerical treatment, International Series of Numerical Mathematics (ISNM) 120 (1995).

[21] W.L. Meyer, W.A. Bell and B.T. Zinn, Boundary integral solutions of three dimensional acoustic radiation problems, Journal Sound and Vibration 59(2) (1978), 245-262. 

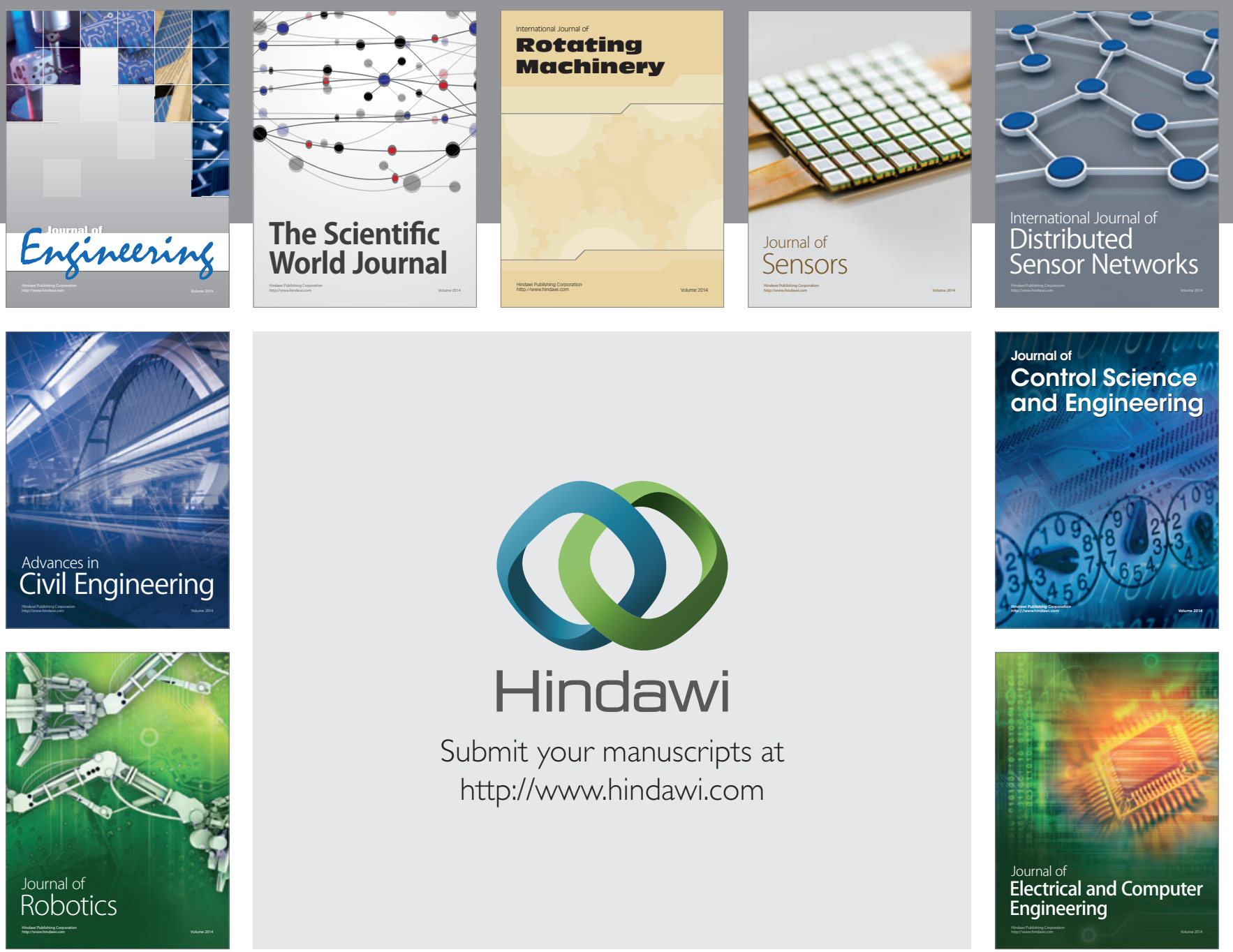

Submit your manuscripts at

http://www.hindawi.com
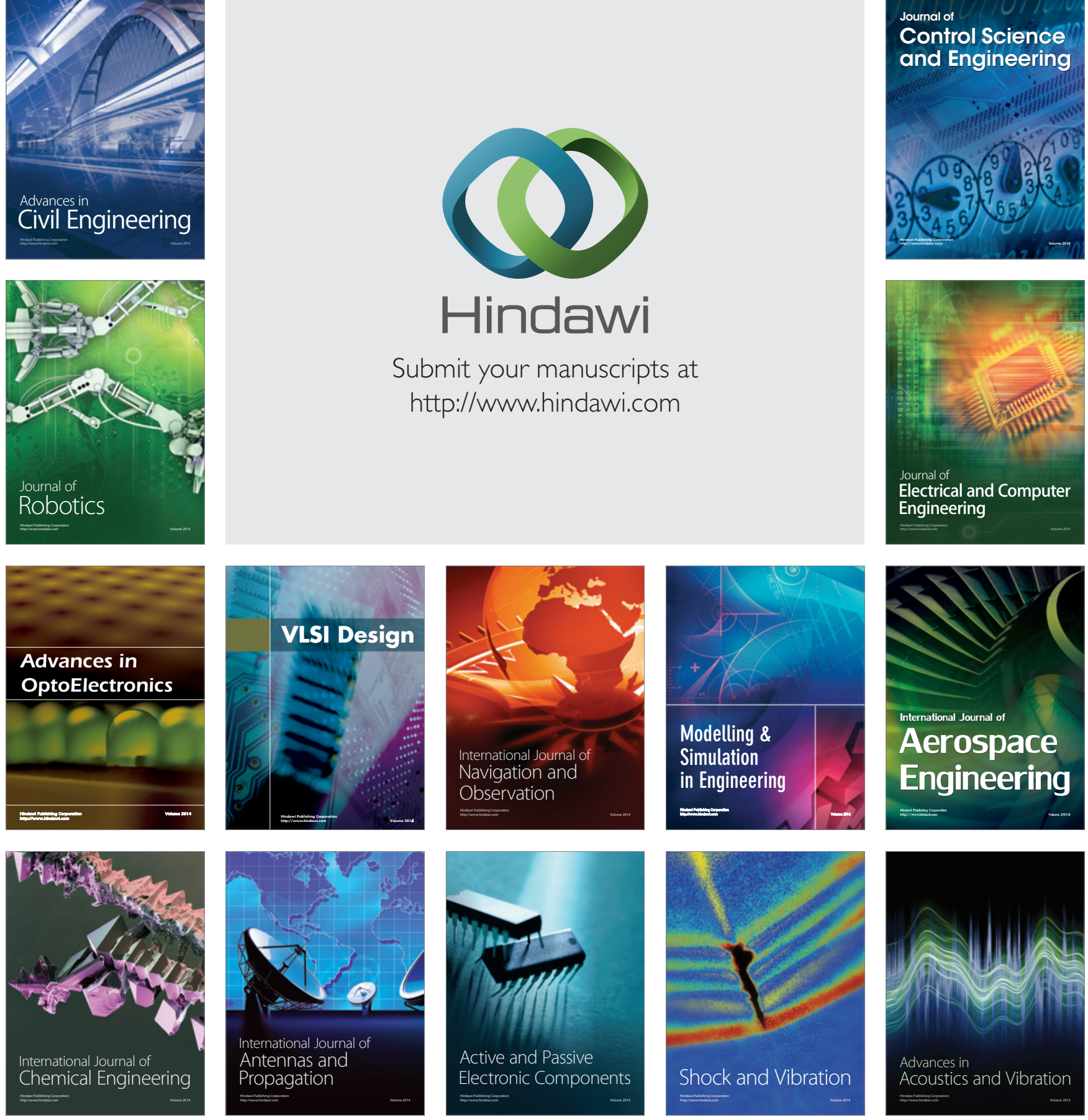